\title{
Activation of Sonic hedgehog signal by Purmorphamine, in a mouse model of Parkinson's disease, protects dopaminergic neurons and attenuates inflammatory response by mediating PI3K/AKt signaling pathway
}

\author{
SHUAI SHAO ${ }^{1,2^{*}}$, GUANG-LIANG WANG ${ }^{3 *}$, CESPUGLIO RAYMOND ${ }^{4}$, \\ XUE-HUA DENG ${ }^{1,5}$, XIAO-LAN ZHU ${ }^{1}$, DI WANG ${ }^{6}$ and LE-PENG HONG ${ }^{1}$ \\ ${ }^{1}$ Department of Anatomy, Guangzhou Medical University, Guangzhou, Guangdong 511436; \\ ${ }^{2}$ Reproductive Medicine Center, Jingmen No. 2 People's Hospital, Jingmen, Hubei 448000; \\ ${ }^{3}$ Department of Histology and Embryology, Guangzhou Medical University, Guangzhou, Guangdong 511436, P.R. China; \\ ${ }^{4}$ Neuroscience Research Center of Lyon (CRNL), Neurochem, Claude Bernard University, F-69373 Lyon, France; \\ ${ }^{5}$ Department of Anatomy, Medical College of Shaoguan University, Shaoguan, Guangdong 512026; \\ ${ }^{6}$ The Second Affiliated Hospital of Guangzhou Medical University, Guangzhou, Guangdong 511436, P.R. China
}

Received December 1, 2016; Accepted May 31, 2017

DOI: $10.3892 / \mathrm{mmr} .2017 .6751$

\begin{abstract}
In Parkinson's disease (PD), microglial activation-mediated neuroinflammation is associated with dopaminergic neurons degeneration in the substantia nigra pars compacta. Previous studies that have investigated this neurodegenerative disease have reported that the Sonic hedgehog (SHH) signaling pathway, through inhibiting the inflammatory processes, exerts a beneficial neuroprotective effect. However, the mechanisms underlying the anti-inflammatory and neuroprotective effects of this signaling pathway remain poorly understood. The present study aimed to further investigate these mechanisms in vitro and in vivo. At first, BV2 microglial cells treated with lipopolysaccharide (LPS) were used to induce an inflammatory response. It was observed that the activation of SHH signaling by Purmorphamine attenuated the LPS-induced inflammatory response, increased the expression of transforming growth factor- $\beta 1$ through the phosphatidylinositol 3-kinase (PI3K)/AKT serine/threonine kinase (Akt) intracellular signaling pathway and inhibited nuclear receptor subfamily 4 group A member 2, independently of the PI3K/Akt signaling pathway. Furthermore, the blockade of the PI3K/Akt signaling pathway by intranasal administration
\end{abstract}

Correspondence to: Professor Le-Peng Hong, Department of Anatomy, Guangzhou Medical University, Guangzhou, Guangdong 511436, P.R. China

E-mail: honglepeng@163.com

*Contributed equally

Key words: Sonic hedgehog, PI3K/Akt, BV2 cells, mouse, MPTP, Parkinson's disease, dopaminergic neurons, inflammation of LY294002, significantly reduced the SHH-associated neuroprotective effects on dopaminergic neurons, improved motor functions, and increased the microglial activation and inflammatory response in a mouse model of PD induced using 1-methyl-4-phenyl-1,2,3,6-tetrahydropyridine. In conclusion, the data of the present study reported that anti-inflammatory and neuroprotective effects can be obtained in BV2 microglial cells and in a mouse model of PD by successive activation of the SHH and PI3K/Akt signaling pathways.

\section{Introduction}

Parkinson's disease (PD) is the second most common neurodegenerative disease characterized by a selective loss of DA neurons in the substantia nigra pars compacta (SNpc) (1). Such a loss produces the depletion of striatal dopamine (DA) which leads to movement disorders such as resting tremor, rigidity, bradykinesia and postural instability (2). Despite the various researches conducted in this field, the precise etiology of PD still remains unclear. Some evidences indicate that the neuroinflammation mediated by microglial activation may play an important role in PD pathogenesis and notably in the degeneration of the SNpc dopaminergic neurons $(3,4)$. Once activated, microglia includes classically activated M1 phenotype (pro-inflammatory function) and also, alternatively, activated M2 phenotype (anti-inflammatory function) $(5,6)$. Microglial activation is believed to lead to neuroinflammation by production of pro-inflammatory cytokines such as interleukin-1 (IL-1), IL-6, tumour necrosis factor- $\alpha$ (TNF- $\alpha$ ), nitric oxide (NO) and prostaglandin E2 (PGE2) (7), which contribute to dopaminergic neuronal death in PD.

The Sonic hedgehog (SHH) diffusible protein, a member of the hedgehog family, become active in binding Patched receptors (Ptch). This binding relieves Ptch-mediated inhibition exerted on Smoothened (Smo) receptor (a key transducer of 
the Hedgehog, HH, signaling pathway). Subsequently, the Smo receptor promotes the transcription factor 'Glioma-associated oncogene' (Gli) which translocate to the nucleus and regulates the transcription and expression of target genes $(8,9)$. $\mathrm{SHH}$ signaling pathway is also activated in brain injuries, neurodegenerative diseases and neurogenesis processes taking place in adult (10-13). SHH signaling can also protect dopaminergic cells from MPP (+) toxicity in vitro and improve nigrostriatal pathway by restoring tyrosine hydroxylase $(\mathrm{TH})$ positivity in vivo (14). In this frame, a recent report suggests that $\mathrm{SHH}-\mathrm{N}$ overexpression can improve the motor function in PD model, restore the nigrostriatal pathway and reduce the loss of DA neurons in vivo (15). Finally, various other reports indicate that PI3K/Akt pathway, related with inflammation, is impaired in PD animal models $(16,17)$. In this sense, Fasudil, a vasodilatator and a potent Rho-kinase inhibitor, is suggested for protecting dopaminergic neurons through the inflammatory inhibition running through the activation of PI3K/Akt signaling pathway (18). Further evidences also suggest that SHH pathway may protects cortical neurons and astrocytes from oxidative stress by activating the PI3K/Akt pathway $(19,20)$. To date, despite the various approaches mentionned above, the precise mechanisms involving a neuroprotective effects of the $\mathrm{SHH}$ signaling pathway still remain unclear.

In the present study, by way of in vitro (LPS-treated BV2 microglial cells) and in vivo (MPTP-induced mouse model of Parkinson disease) approaches, we demonstrate that the SHH signaling through the PI3K/AKt pathway is capable to: Attenuate the inflammatory response, inhibit the microglial activation, protect dopaminergic neurons and reduce behavioral impairments.

\section{Materials and methods}

Materials. The following reagents were used in the present study: Dulbecco's modified Eagle's medium (DMEM) and fetal bovine serum (FBS), from Gibco (Grand Island, NY, USA); TH antibody, p-AKt antibody and AKt antibody, from Abcam (Cambridge, UK); Ionized calcium binding adaptor molecule 1 (Iba1) antibody, from Wako (Osaka, Japan); $\beta$-actin antibody, HRP-conjugated goat polyclonal anti-rabbit IgG antibody, Purmorphamine and Cyclopamine, from Santa Cruz Biotechnology, Inc. (Santa Cruz, CA, USA); LPS and MPTP, from Sigma-Aldrich (St. Louis, MO, USA); LY294002, from Cell Signaling Technology, Inc. (Danvers, MA, USA).

Cell culture. The murine BV2 microglial cell line was grown in DMEM supplemented with 10\% FBS (Gibco), $100 \mathrm{U} / \mathrm{ml}$ penicillin, and $100 \mathrm{mg} / \mathrm{ml}$ (Sigma, St. Louis, MO, USA). In a humidified $5 \% \mathrm{CO}_{2}$ incubator maintained at $37^{\circ} \mathrm{C}$, streptomycin and the culture medium were renewed every day. Cells were plated at $5 \times 10^{5}$ concentration and grown for $24 \mathrm{~h}$ prior to the experiments.

In vitro treatments. Dishes of cultured BV2 cells were randomly divided into six groups: Including: i) Control group, ii) LPS group, iii) PM+LPS group, iv) Cyclopamine+PM+LPS group, v) LY294002+PM+LPS group and vi) LY294002+LPS group. For LPS group: LPS $(1 \mu \mathrm{g} / \mathrm{ml})$ was employed during $24 \mathrm{~h}$ to obtain an inflammatory response with BV2 cells. For
PM+LPS group: Purmorphamine (PM, $1.5 \mu \mathrm{mol} / \mathrm{l}$ ) was used to activate the SHH pathway in BV2 cells $24 \mathrm{~h}$ before LPS treatment; For Cyclopamine+PM+LPS group: BV2 cells were pretreated with a specific SHH signal inhibitor (Cyclopamine) to further explore the role of PI3K/Akt pathway on the effects of SHH pathway; in this respect, Cyclopamine $(20 \mu \mathrm{mol} / \mathrm{l})$ was administered to block the SHH pathway (1 h before PM treatment); then, PM was used to treat BV2 cells for $24 \mathrm{~h}$; then after, a LPS treatment was applied. For LY294002+PM+LPS group: A selective inhibitor of PI3K/AKt, LY294002 $(20 \mu \mathrm{mol} / \mathrm{l})$ was used during $30 \mathrm{~min}$ in order to block PI3K/Akt pathway before PM treatment; then PM was used to treat BV2 cells for $24 \mathrm{~h}$; this pharmacological situation was completed with a LPS treatment. For LY294002+LPS group: LY294002 was used to treat BV2 cells without PM treatment; then after, a LPS treatment was applied.

In vivo treatment. All animal studies were approved by the Institutional Animal Care and Use Committee at Guangzhou Medical University. Male C57BL/6 mice (8-10 weeks, 22-25 g) were housed under a 12-h light/dark cycle with free access to food and water. All animals were randomly divided into five group, including: i) Control $(n=10)$; ii) MPTP $(n=10)$; iii) PM+MPTP (n=11, PM+MPTP); iv) LY294002+PM+MPTP (n=11, LY+PM+MPTP); and v) LY294002+MPTP $(n=11$, LY+MP). For the MPTP group, mice received four intraperitoneally (i.p.) injections of MPTP $(20 \mathrm{mg} / \mathrm{kg})$ in a $2 \mathrm{~h}$ interval (21); For the PM+MPTP group, mice received i.p. injection of PM $(1 \mathrm{mg} / \mathrm{kg}) 24 \mathrm{~h}$ before the first MPTP injection; For the LY+PM+MPTP group, $30 \mu \mathrm{l}$ of LY294002 $(5 \mathrm{mg} / \mathrm{ml})$ were administered intranasally $1 \mathrm{~h}$ before PM treatment; then after, PM was administered i.p. and, 24 h later, MPTP was given. For the LY294002+MPTP group, LY294002 was administered intranasally and MPTP was injected four times.

Behavioral tests. Traction behavior (TR) test was described previously (22). The stainless steel bar (diameter: $1.5 \mathrm{~mm}$, $25 \mathrm{~cm}$ long) was fixed $30 \mathrm{~cm}$ over ground. Each animal was hanged by its forelimbs and left on the bar. The time lapse before the fall down was checked for each animal. For the score of TR, the following standards were employed: $0-4$ sec $=0$; 5-9 $\mathrm{sec}=1 ; 10-14 \mathrm{sec}=2 ; 15-19 \mathrm{sec}=3 ; 20-24 \mathrm{sec}=4 ; 25-29=5$; over $30=6$. Each animal was submitted three times to the test with an interval of $1 \mathrm{~min}$ between each trial. The general mean of the trials was taken.

Quantitative PCR ( $q P C R$ ). For qPCR analysis, total RNA was isolated from BV2 cells and from the brain substantia nigra by TRIzol reagent (Takara Bio, Inc., Otsu, Japan). Total RNA $(1 \mu \mathrm{g})$ was reverse transcribed into cDNA using PrimerScript $^{\mathrm{TM}}$ RT reagent kit (Takara Bio, Inc.). The mRNA expression levels were measured to use $\mathrm{SYBR}^{\circledR}$ Premix Ex Tag $^{\mathrm{TM}}$ and primers. The sequences for $\mathrm{qPCR}$ promers were as follows: IL-1 $\beta$ forward, 5'-TGCCACCTTTTGACAGTG ATG-3'; reverse, 5'-GGAAG for each GTCCACGGGAAA GAC-3'; TNF- $\alpha$ forward, 5'-ATGGCCTCCCTCTCATCA GT-3'; reverse, 5'-ATAGCAAATCGGCTGACGGT-3'; Nurr1 forward, 5'-AAGACCTTCTCCCAAGCACG-3'; reverse, 5'-GAACTGGACACTTCAACCAGC-3'; TGF- $\beta 1$ forward, 5'-GGTCCTTGCCCTCTACAACC-3'; reverse, 5'-CCACGT 
AGTAGACGATGGGC-3'; $\beta$-actin forward, 5'-GTTACAGGA AGTCCCTCACCC-3'; reverse, 5'-CAGAAGCAATGCTGT CACCTT-3'. Cycling condition included one cycle at $95^{\circ} \mathrm{C}$ for $5 \mathrm{sec}, 30$ cycles at $95^{\circ} \mathrm{C}$ for $30 \mathrm{sec}$ and $60^{\circ} \mathrm{C}$ for $1 \mathrm{~min}$, followed by one cycle $95^{\circ} \mathrm{C}$ for $15 \mathrm{sec}, 60^{\circ} \mathrm{C}$ for $1 \mathrm{~min}$ and $95^{\circ} \mathrm{C}$ for $15 \mathrm{sec} . \beta$-actin was used as an internal control and the transcript levels are expressed as $2^{-\Delta \Delta \mathrm{Cq}}$ values.

Western blotting. BV2 cells and substantia nigra were lysed in ice cold lysis buffer (1X PBS, 1\% Nonidet P-40, 05\% sodium deoxycholate and $0.1 \%$ SDS; RIPA) containing phosphatase inhibitor protease inhibitor. Lysates (50 $\mu \mathrm{g}$ protein) from each sample were resolved on SDS $10 \%$ polyacrylamide gel (10\% PAGE) and electrotransferred to PVDF membrane. The membrane was incubated in 5\% non-fat dry milk to block non-specific antibody binding site and then incubated overnight at $4^{\circ} \mathrm{C}$ with rabbit anti-p-AKt $(1: 1,000)$, rabbit anti-AKt $(1: 1,000)$, rabbit anti-TH $(1: 200)$, rabbit anti-Iba1 $(1 \mu \mathrm{g} / \mathrm{ml})$, rabbit anti- $\beta$-actin $(1: 1,000)$. After washing in TBST $(0.01 \mathrm{M}$ TBS and $0.1 \%$ Tween-20), membranes were incubated with horseradish peroxidase-conjugated secondary antibodies (goat anti-rabbit immunoglobulin $\mathrm{G}$ [IgG], 1:1,000) for $2 \mathrm{~h}$. This step was followed by a washing in TBST and protein visualized. In this respect, chemiluminescence (ECL) reagents were employed.

Immunohistochemistry. Mice were anesthetized and perfused transcardially with phosphate-buffered saline (PBS) followed by a $4 \%$ fresh paraformaldehyde (PFA) solution ( $\mathrm{pH} 7.4)$. Brains were removed and kept in $4 \%$ PFA overnigth at $4^{\circ} \mathrm{C}$. Then after, they were inserted in a $30 \%$ sucrose solution for $48 \mathrm{~h}$ at $4^{\circ} \mathrm{C}$. Afterwards, brains were cutted (slices of $30 \mathrm{~m}$ depth) with a freezing microtome (Leica, Germany) and incubated during $30 \mathrm{~min}$ in $3 \% \mathrm{H}_{2} \mathrm{O}_{2}$ solution. Then, slices were rinsed three times and during $5 \mathrm{~min}$ in PBST (0.01 M PBS and 0.3\% Triton-X 100) and incubated with a $5 \%$ normal goat serum at room temperature for $1 \mathrm{~h}$. Brain section were then incubated with polyclonal rabbit anti-TH (1:750; cat. no. ab112; Abcam), Ibal $(0.5 \mu \mathrm{g} / \mathrm{ml}$; Wako) overnight at $4^{\circ} \mathrm{C}$. The next day, the sections were washed with PBST three times for $5 \mathrm{~min}$ and incubated with corresponding secondary antibodies at room temperature for $2 \mathrm{~h}$. They were washed again in PBS and subsequently incubated with streptavidin-peroxidase for $30 \mathrm{~min}$. After a new wash in PBS ( $3 \times 5$ min.), they were incubated with a DAB chromogenic substrate (Fuzhou Maixin Biotech Co., Ltd. (Fuzhou, China) for $5 \mathrm{~min}$ and then after washed again in distilled water. Afterwards, brain sections were mounted on gelatin-coated slides, air-dried, dehydrated, and covered with a glass plate. Brain sections were examined using a light-field microscope. Finally, $\mathrm{TH}^{+}$cells were identified with the Image-Pro Plus software and visually counted.

Statistical analysis. The Statistical Package for the Social Sciences (SPSS version 13.0) was used for the statistical analyses. All data were expressed as mean \pm standard deviation (SD) for three independent experiments, at least. The analysis of variance (ANOVA) was performed for all tests, followed by post hoc Fisher's LSD multiple comparison test. $\mathrm{P}<0.05$ was considered to indicate a statistically significant difference.

\section{Results}

SHH signaling pathway activates the PI3K/Akt signaling pathway. Data obtained indicate that LPS-induced inflammatory response in BV2 microglial cells produces a significant reduction in $\mathrm{p}$-AKt protein content when compared with the saline group of animals. This effect, however, does not reach significance vs. total AKt proteins. Activation of SHH pathway by PM enhances the expression of p-AKt protein, which can be reversed by the SHH pathway inhibitor cyclopamine. No change are observed in total AKt proteins after PM and cyclopamine treatments (Fig. 1A, D and G). Western blot analysis shows that treatment of BV2 cells with LY294002 suppresses the SHH signaling mediated by the AKt activation and accelerates the LPS mediated AKt signalling suppression (Fig. 1B, $\mathrm{E}$ and $\mathrm{H})$.

We also checked whether the SHH pathway regulated the expression of $\mathrm{p}$-AKt protein in the area of $\mathrm{SNpc}$ in mice model of PD. As shown in Fig. 1C, F and I, MPTP causes a significant decrease in the level of p-AKt, activation of $\mathrm{SHH}$ signaling and a significant increase in the level of $\mathrm{p}-\mathrm{AKt}$ protein when compared with MPTP-treated group of animals. To analyze how are combined PI3K/Akt and SHH signalings, we employed an intranasal administration of LY294002 to inhibit PI3K/Akt component. Western blot analysis shows that intranasal administration of LY294002 abrogated the PM-induced restoration of $\mathrm{p}$-AKt and accelerates MPTP mediated P-AKt inhibition. These results indicate that inflammatory response may inhibit the PI3K/Akt signaling while the SHH pathway may activate the PI3K/Akt signaling in vitro and in vivo.

SHH signaling pathway inhibits microglial activation through the PI3K/Akt signaling pathway. As shown in Fig. 2A, a large number of Iba1-positive microglial cells characterized by amoeboid shape with larger cell bodies and shorter processes are observed in MPTP group compared with saline group. In Mice treated with PM and MPTP, small microglial cell with reduced cell bodies, ramifications and thin processes are also observed. By contrast, numerous activated microglial cells are accompanied by enlarged cell bodies and thicker processes in LY+PM+MPTP group. Morphology of Iba1-positive microglial cells in LY+PM+MPTP group are similar to MPTP and LY+MPTP groups.

Western blot reveals that LY294002 blocked the effect of PM treatment on microglial cells: The Ibal protein is significantly increased in the MPTP group. This effect, however, is also observed when the PM group is inhibited by LY294002. LY294002 increases the expression of Iba1 protein compared with MPTP group alone (Fig. 2B and C). These results indicate that $\mathrm{SHH}-\mathrm{PI} 3 \mathrm{~K} / \mathrm{Akt}$ relayed signaling inhibit activation of the microglia.

SHH signaling mediated anti-inflammatory effects through the PI3K/Akt signaling pathway. To check this aspect, SNpc tissue homogenate from our five group of animals were performed. As shown in Fig. 3A and B PM pretreatment decreases the expression of IL- $1 \beta$ and TNF- $\alpha$ mRNA. This aspect is reversed by LY294002. Moreover, the inhibition of the PI3K/Akt pathway with LY294002 in LPS treated 
A

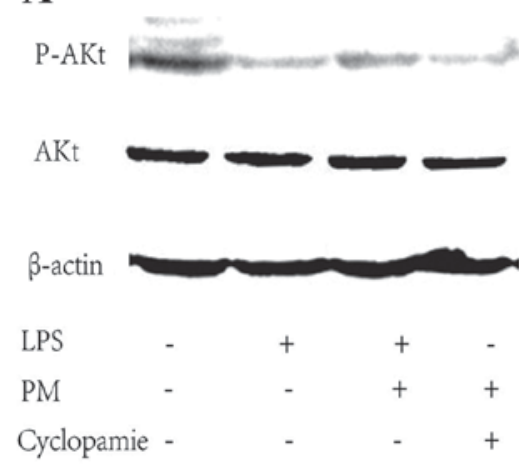

D

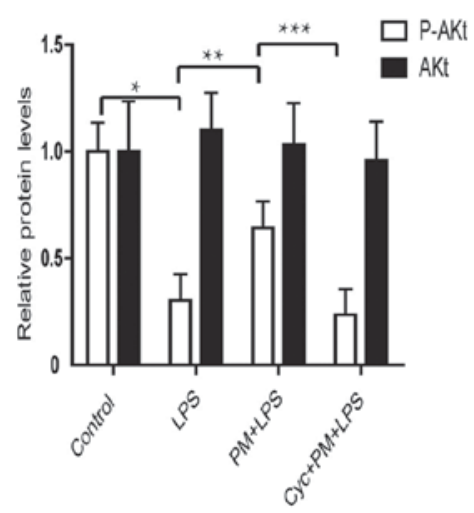

G

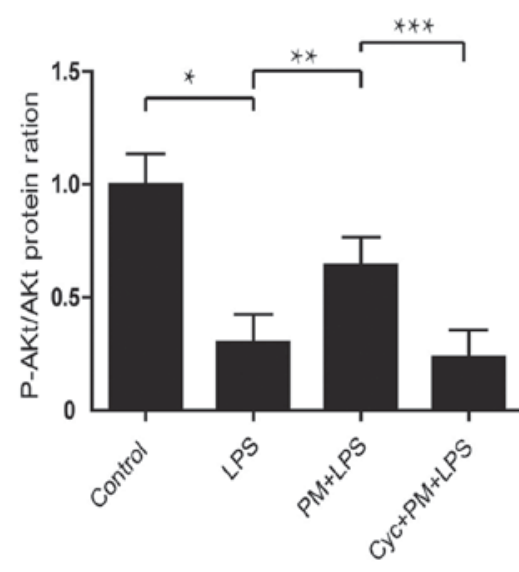

B

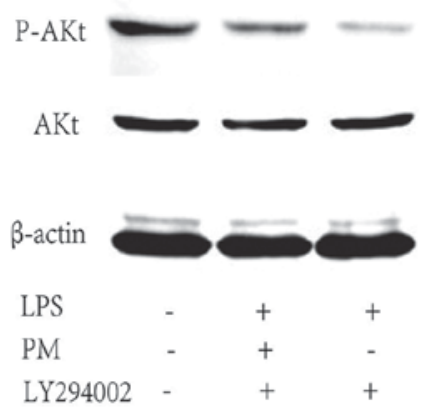

C

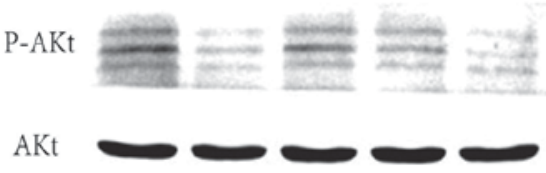

$\beta$-actin

$\begin{array}{llllll}\text { MPTP } & - & + & + & + & + \\ \text { PM } & - & - & + & + & -\end{array}$

LY294002 - $\quad-\quad+\quad+\quad+$

E

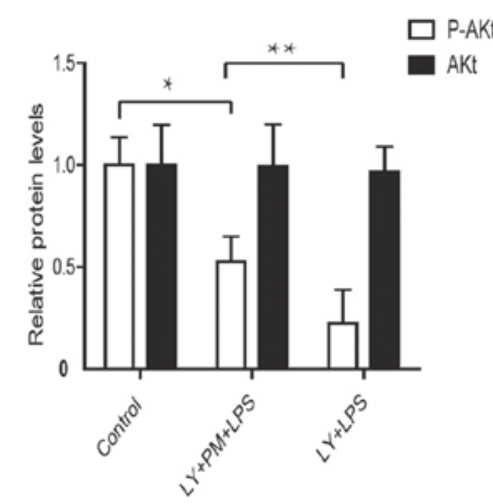

H

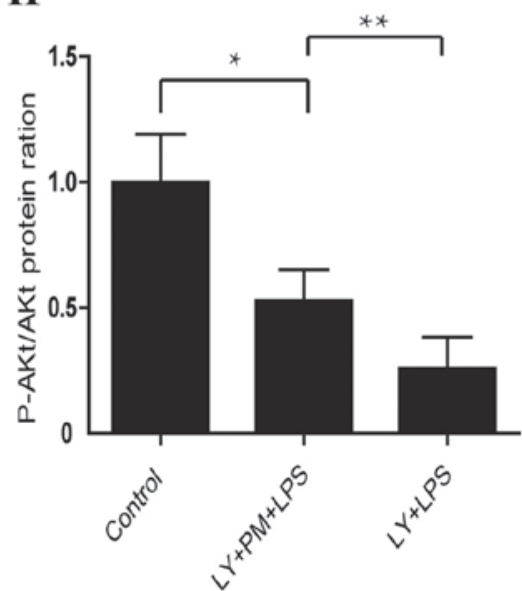

F

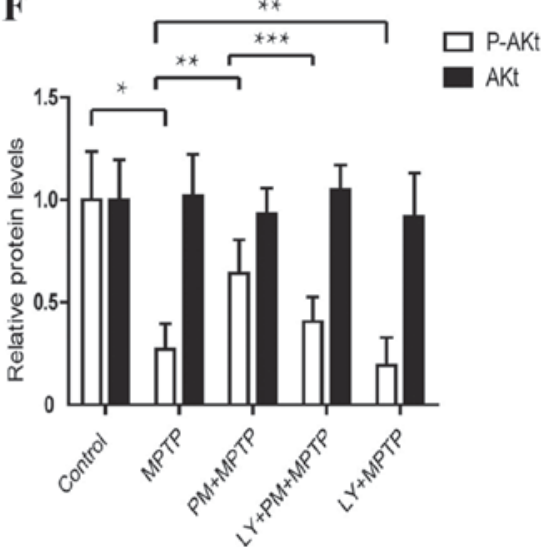

I

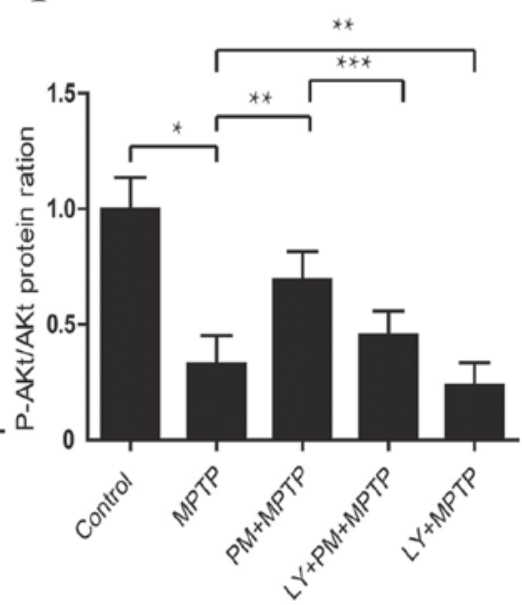

Figure 1. Purmorphamine (PM) and LY294002 respectively activates and inhibits Sonic hedgehog (SHH) signaling by way of PI3K/AKt pathway either in vitro or in vivo. (A) BV2 cells successive treatments were as it follows: At first, Cyclopamine ( $20 \mu \mathrm{mol} / \mathrm{l})$ was given; $1 \mathrm{~h}$ later PM (1.5 $\mu$ mol) was added; after a $24 \mathrm{~h}$ time lapse LPS $(1 \mu \mathrm{g} / \mathrm{ml})$ was applied; $24 \mathrm{~h}$ after this last treatment AKt phosphorylation was checked by Western blot and $\beta$-actin was used as an internal control. (B) BV2 cells successive treatment were as it follows: At first, LY294002 (20 $\mu \mathrm{mol} / \mathrm{l}) ; 0.5 \mathrm{~h}$ later a BV2 plot received PM (1.5 $\mu$ mol) while a second plot did not received this substance; LPS $(1 \mu \mathrm{g} / \mathrm{ml})$ was finally added to both plots for a $24 \mathrm{~h}$ time lapse. After LPS treatment AKt phosphorylation was assessed by Western blot. (C) C57/BL6 mouse received the following successive treatments: At first, LY294002 (5 mg/ml, $30 \mu \mathrm{l})$ in nasal cavity, or saline $0.9 \%$ i.p., were given; $1 \mathrm{~h}$ later, PM $(1 \mathrm{mg} / \mathrm{kg})$ was administered; finally, $24 \mathrm{~h}$ later, four injections of MPTP $(20 \mathrm{mg} / \mathrm{kg}$, i.p.) were achieved within a practical time lapse of $2 \mathrm{~h}$. Mice were then sacrificed and the entire SNpc collected to achieve AKt phosphorylation by Western blot. (D-F) Relative levels of AKt protein and P-AKt in vivo and in vitro. (G-I) Protein ratio of $\mathrm{P}-\mathrm{AKt} / \mathrm{AKt}$ in vivo and in vitro. ${ }^{*} \mathrm{P}<0.01$ compared with control group; ${ }^{* *} \mathrm{P}<0.01$ compared with $\mathrm{LPS}$ or MPTP group or LY+PM+LPS; ${ }^{* * *} \mathrm{P}<0.01$ compared with PM+LPS or PM+MPTP group; ${ }^{*} \mathrm{P}<0.01$ compared with LY+PM+LPS group. PM, Purmorphamine; LY, LY294002; LPS, lipopolysaccharide; i.p., intraperitoneally injections; SNpc, substantia nigra pars compacta.

group increases the expression of IL- $1 \beta$ and TNF- $\alpha$ mRNA compared with LPS treated group.

We also investigated the role of PI3K/Akt signaling in vitro. As expected, the results obtained indicate that
SHH pathway mediates the expression of pro-inflammatory factors IL-1 $\beta$, TNF- $\alpha$ mRNA through the PI3K/Akt signaling (Fig. $3 \mathrm{C}$ and D). Moreover, the inhibition of the PI3K/Akt signaling with LY294002 reverses the increased 
A
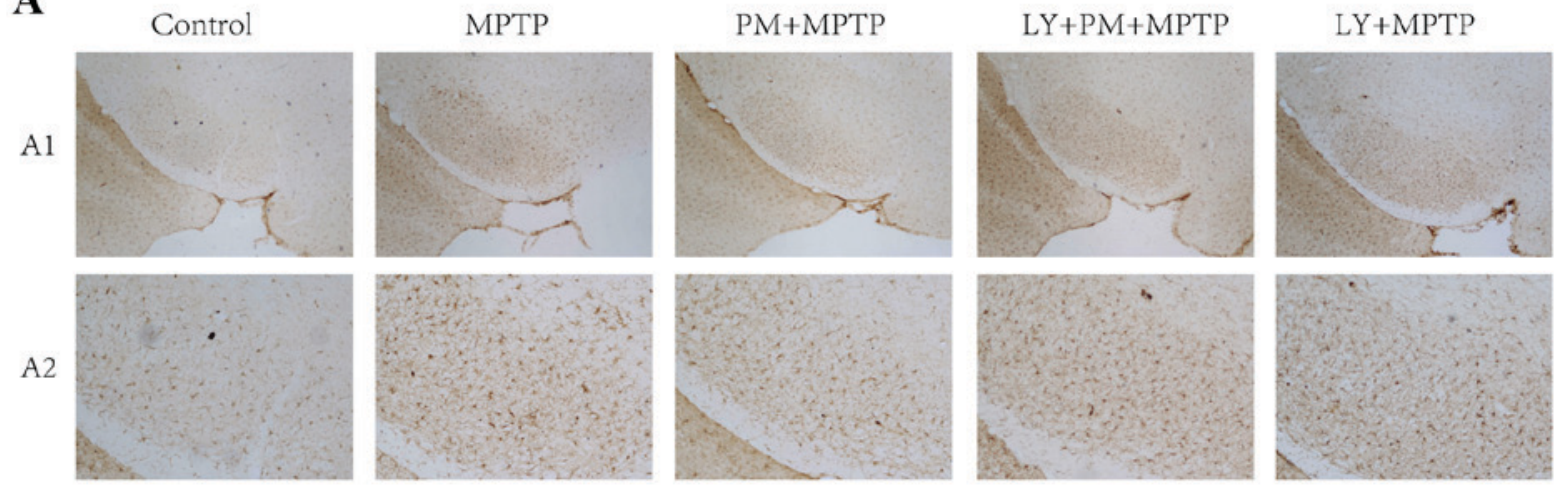

A3
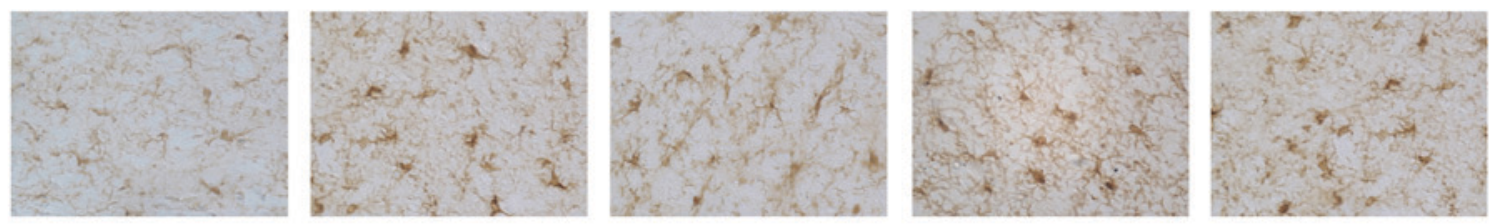

B

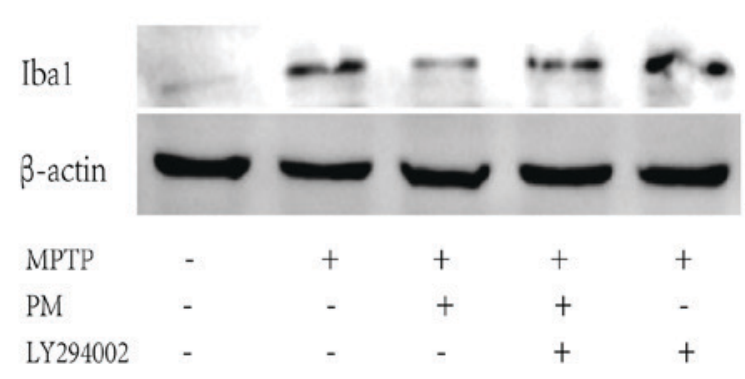

C

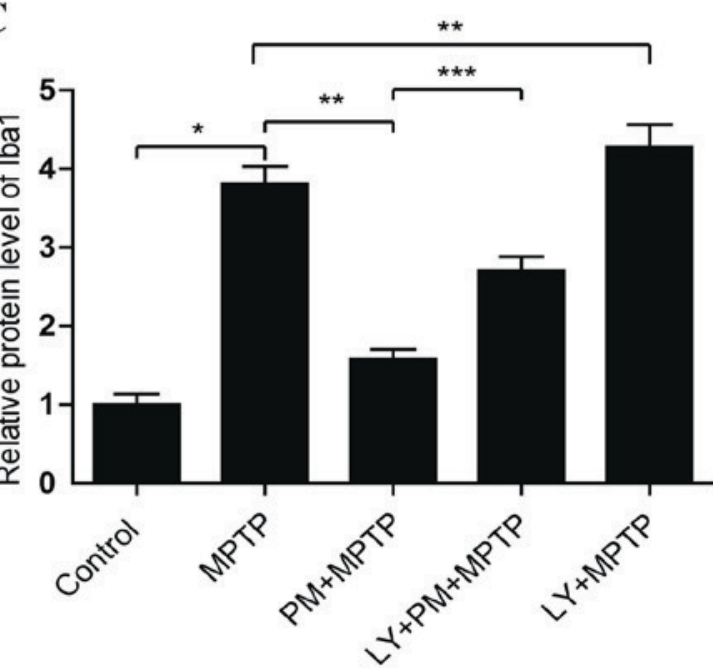

Figure 2. SHH signaling inhibits microglia activation through PI3K/Akt pathway. (A) We performed immunohistochemical staining with Iba1 to visualize the activation of microglia in SNpc of five mice groups: i) Control group; ii) MPTP group; iii) PM+MPTP group; iv) LY+PM+MPTP group; and v) LY+MPTP group. A1 scale bar $=500 \mu \mathrm{m}$; A2 scale bar=200 $\mu \mathrm{m}$; A3 scale bar=50 $\mu \mathrm{m}$. (B) The Iba1 protein in SNpc were determined using western blotting and analysis of Iba1. (C) Relative level of Iba1 protein: " $\mathrm{P}<0.01$ compared with control group; ${ }^{* *} \mathrm{P}<0.01$ compared with MPTP group; ${ }^{* * * *} \mathrm{P}<0.01$ compared with PM+MPTP group. SHH, Sonic hedgehog; Iba1, Ionized calcium binding adaptor molecule 1; LY, LY294002.

expression of the anti-inflammatory factor TGF- $\beta 1$, induced by PM (Fig. 3E). Finally, it appears that SHH signaling inhibits Nurr1 independently of the PI3K/AKt pathway (Fig. 3F).

SHH signaling mediated neuroprotection through the PI3K/Akt signaling pathway. TH immunohistochemistry reveals a significant cell reduction in MPTP group compared with the saline one. Treatment with PM protects dopaminergic neurons of the $\mathrm{SNpc}$ and enhances their rate of survival. However, pretreatment with LY294002 abolishes the neuroprotection exerted by PM in the model of PD and also decreases the number of TH-positive neurons compared with MPTP group (Fig. 4A and B). Results established with the western blotting are similar to those obtained with immunohistochemistry. They show that MPTP causes a significant decrease in the level of TH protein. PM treatment accelerates the restoration $\mathrm{TH}$ protein level, but the increase in TH protein level in the PM group of animals is inhibited by the administration of LY294002. This last substance when administered alone, reduces also the expression of $\mathrm{TH}$ protein when compared with the MPTP group of animals (Fig. 4C and D).

SHH signaling pathway induced behavioral change through the PI3K/Akt signaling pathway in MPTP-treated mice. With TR test, mice treated with MPTP spend significantly less time on the steel compared with those treated with saline (Fig. 5). This result indicates an impairment of motor balance and coordination. However, PM treatment rescued the MPTP-induced motor deficit, as reflected by the marked increase in the time spent on the steel. This effect can be abrogated by LY294002. Finally, TR test score was not statistically different between MPTP and LY+MPTP groups. These data support clearly that SHH-mediated motor function improvements are dependent on PI3K/Akt signaling pathway. 
A

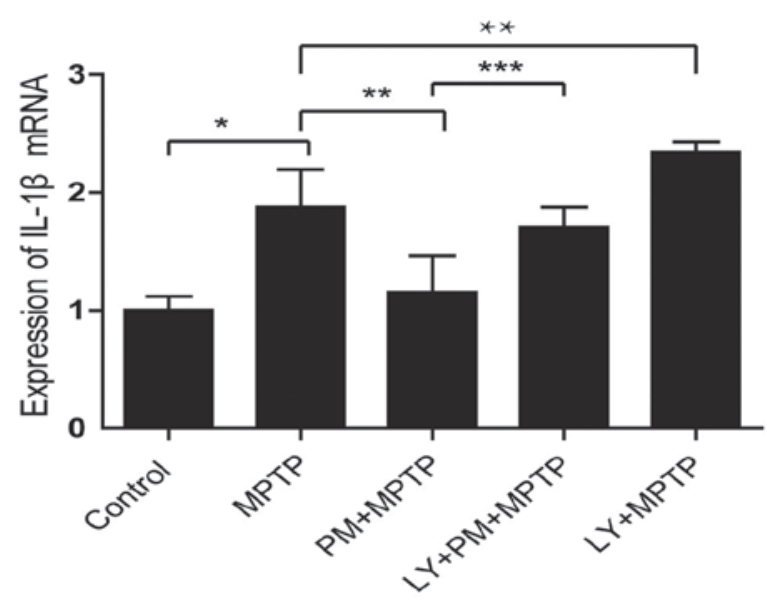

C

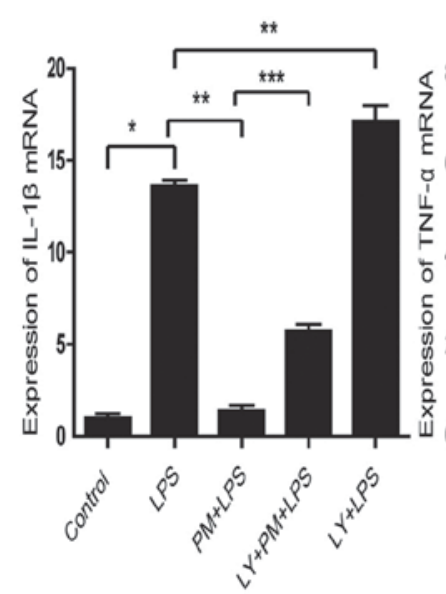

D

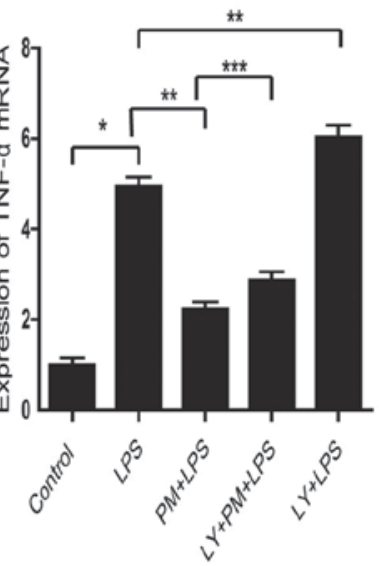

B

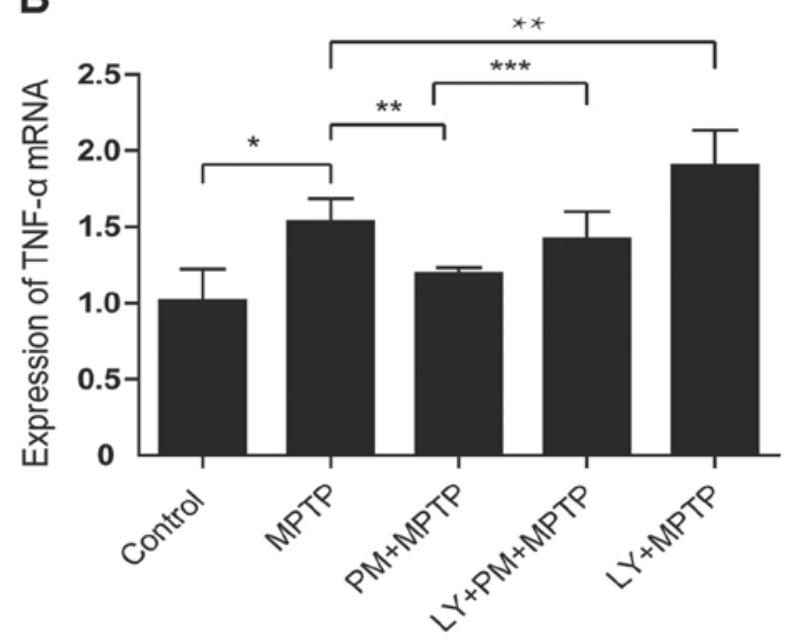

E

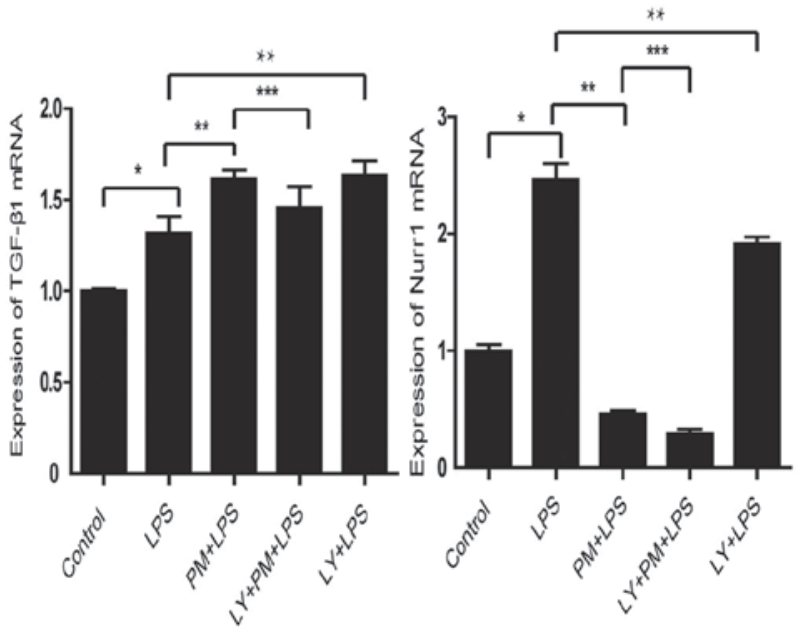

Figure 3. SHH signaling mediates anti-inflammatory effects through PI3K/Akt pathway. (A and B) Expression of IL-1 $\beta$ and TNF- $\alpha$ mRNA in SNpc by quantitative real time PCR analysis. (C-F) Expression of IL- $1 \beta$, TNF- $\alpha$, TGF- $\beta, 1$ and Nurr1 mRNA in BV2 cells by quantitative real time PCR analysis. "P<0.01 compared with control group; ${ }^{* *} \mathrm{P}<0.01$ compared with LPS or MPTP group; ${ }^{* * *} \mathrm{P}<0.01$ compared with PM+LPS or PM+MPTP group.

\section{Discussion}

In the present study, we further investigated the part played by the SHH signaling in vitro, in using BV2 microglial cells treated with LPS and, in vivo, in using the MPTP to induced the animal model of PD. We found that SHH signaling exerts anti-inflammatory and neuroprotective effects either in vitro or in vivo, at least partly, through PI3K/Akt signaling pathway. We also evidenced that SHH signaling pathway may activate the PI3K/Akt signaling and restore the level of P-AKt which is repressed in neuroinflammatory conditions and in animal models of PD.

Data of literature, in keeping with our results, now point out that neuroinflammation, triggered by microglial activation, may play a central role in the progression of the dopaminergic neurons degeneration in PD pathogenesis $(23,24)$. These data also report that the microglial activation occurs in the early stage of PD pathogenesis and precedes the degeneration of dopaminergic neurons (25). In addition to these aspects, it is further stated that mesencephalic dopaminergic neurons express SHH which is necessary to maintain their structure and function. Different other studies indicate that the loss of SHH signaling conduces to the dopaminergic neuron degeneration and that the administration of exogenous $\mathrm{SHH}$ protein increases the survival rate of these neurons in MPTP or 6-OHDA treated animals (26). Our results are thus in good agreement with previous approaches showing that the microglial activation is linked with the progressive degeneration of the DA neurons in PD pathogenesis (27). Microglial activation may, indeed, exacerbate dopaminergic degeneration in releasing a large number of pro-inflammatory cytokines such as IL-1 $\beta$, IL-6, TNF- $\alpha$, and NO (28).

Beside above considerations, our results also point out that SHH signaling negatively regulated the activation of microglia through PI3K/Akt pathway. A study of literature indicating that SHH signaling can alleviate inflammatory response (29) is in agreement with our results. Moreover, our results also indicate that the SHH signaling is capable to downregulate the 
A

Control

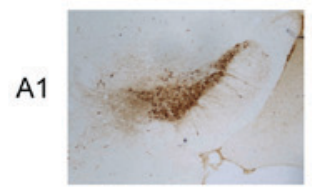

A2

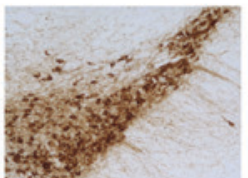

A3

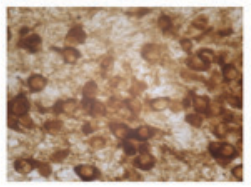

MPTP
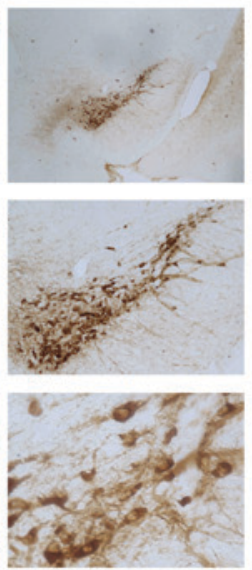

PM+MPTP
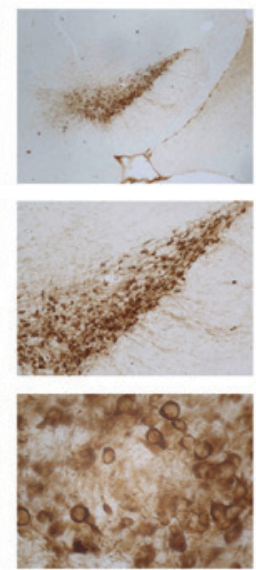

$L Y+P M+M P T P$
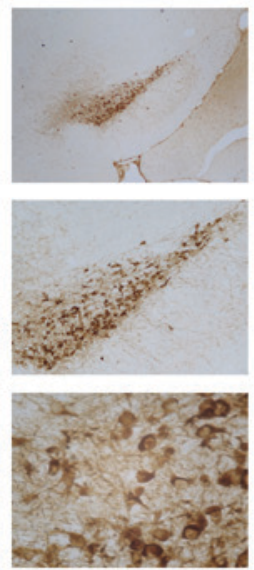

$L Y+M P T P$
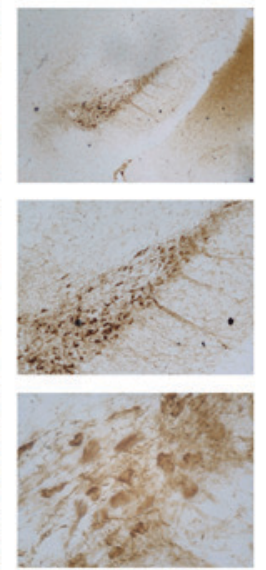

B

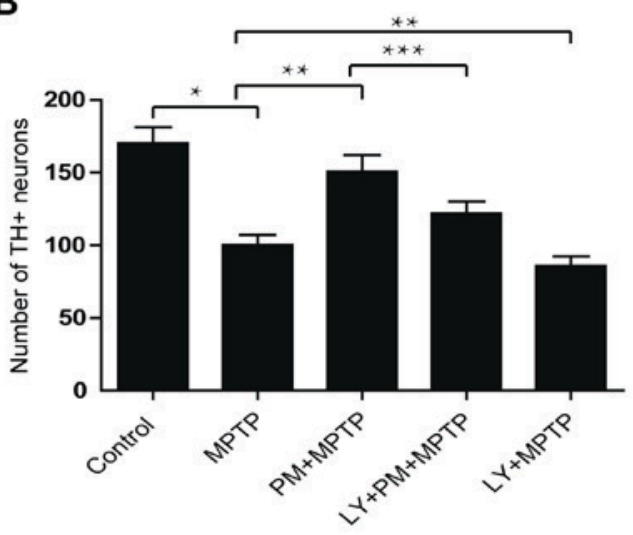

C

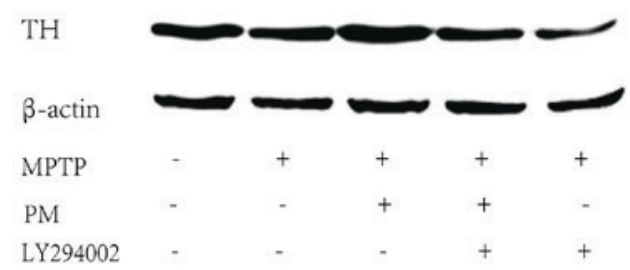

D

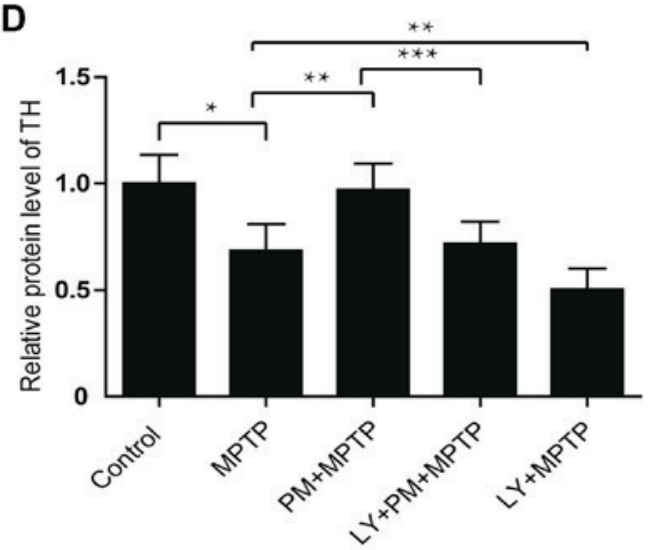

Figure 4. SHH signaling mediates neuroprotection through the PI3K/Akt pathway. (A) Immunohistochemical staining was performed to visualize TH-positive dopaminergic neurons in the SNpc. A1 scale bar $=500 \mu \mathrm{m}$; A2 scale bar=200 $\mu \mathrm{m}$; A3 scale bar=400 $\mu \mathrm{m}$. (B) Number of TH-positive dopaminergic neurons in SNpc. (C) Western blotting and analysis of TH protein. (D) Relative level of TH protein. " $\mathrm{P}<0.01$ compared with control group; ${ }^{* *} \mathrm{P}<0.01$ compared with MPTP group; ${ }^{* * * *} \mathrm{P}<0.01$ compared with PM+MPTP group. TH, Tyrosine hydroxylase.

expression of pro-inflammatory factors like IL-1 $\beta$ or TNF- $\alpha$ and to upregulate the expression of anti-inflammatory factors like TGF- $\beta 1$. Again, they are in keeping with literature data since TGF- $\beta 1$ is known as an anti-inflammatory factor capable to regulate, in animal models of $\mathrm{PD}$, the microglial activation and reduce the neuro-inflammation through PI3K/Akt pathway (30). It is to be noticed, however, that SHH signaling is also capable to inhibit the expression of Nurr1 independently of the PI3K/AKt pathway.
Finally, our data indicating that the activation of the $\mathrm{SHH}$ signaling is essential to prevent, via the PI3K/AKt signaling pathway, the MPTP-induced loss of dopaminergic neurons and the motor deficit are in keeping with those of literature $(15,31)$. They further specify the nature of the processes involved.

Apart from the data discussed above, in the full statement of our results it remains to be further understood: How the LY294002 inhibitor of the PI3K/Akt signaling pathway may downregulate the expression of TGF- $\beta 1$ induced by PM; 


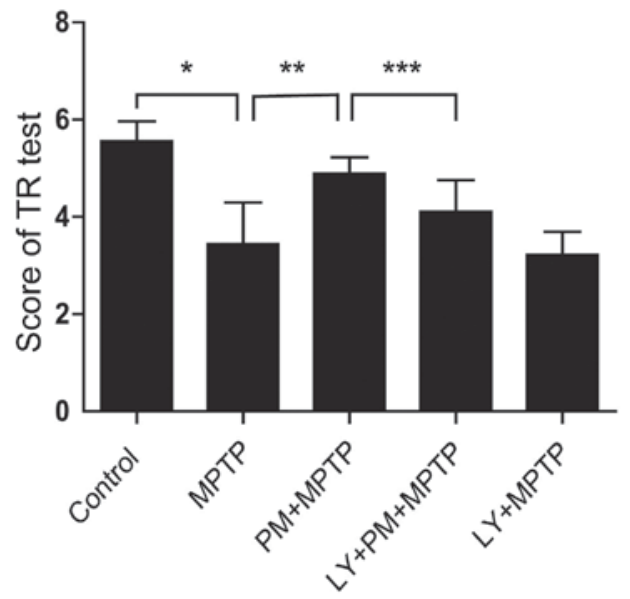

Figure 5. SHH signaling induces behavioral change through PI3K/Akt pathway in MPTP mice model of PD. Traction behavior (TR) test was applied to test the motor function. Scale given in Material and methods was employed to evaluate performances. ${ }^{*} \mathrm{P}<0.01$ compared with control group; ${ }^{* *} \mathrm{P}<0.01$ compared with MPTP group; ${ }^{* * *} \mathrm{P}<0.01$ compared with $\mathrm{PM}+\mathrm{MPTP}$ group. TR, Traction behavior.

how this inhibitor can upregulate the expression of TGF- $\beta 1$ induced by LPS in vitro. Additive experiments still remain necessary to clarify these aspects. Moreover, Nurr1, an orphan nuclear receptor, reported as critical for the development and maintenance of dopaminergic neurons (32), also capable to attenuate the expression of proinflammatory molecules released by microglial (33), appears, according to our results, inhibited by the SHH signaling and independent of the PI3K/AKt signaling pathway. Again, further experiments are still necessary to clarify these last aspects. They are part of our prospects.

In conclusion, our study demonstrates that SHH-PI3K/Akt coupled signalings are capable to protect DA neurons, inhibit microglia activation and improve motor performances. While additive experiments remains, obviously, necessary to clarify the results reported, our research appears promising for a better understanding of the neurodegenerative pathologies.

\section{Acknowledgments}

This study was supported by grants from the Guangdong Provincial Development of Science and Technology (no. 201301) and the Medical Scientific Research Foundation of Guangdong Province (no. A2013239). PR Raymond CESPUGLIO contributed to the manuscript improvement.

\section{References}

1. Mullin S and Schapira AH: Pathogenic mechanisms of neurodegeneration in Parkinson disease. Neurol Clin 33: 1-17, 2015.

2. Berardelli A, Wenning GK, Antonini A, Berg D, Bloem BR, Bonifati V, Brooks D, Burn DJ, Colosimo C, Fanciulli A, et al: EFNS/MDS-ES/ENS [corrected] recommendations for the diagnosis of Parkinson's disease. Eur J Neurol 20: 16-34, 2013.

3. Terada T, Yokokura M, Yoshikawa E, Futatsubashi M, Kono S, Konishi T, Miyajima H, Hashizume T and Ouchi Y: Extrastriatal spreading of microglial activation in Parkinson's disease: A positron emission tomography study. Ann Nucl Med 30: 579-587, 2016.
4. Tanaka S, Ishii A, Ohtaki H, Shioda S, Yoshida T and Numazawa S: Activation of microglia induces symptoms of Parkinson's disease in wild-type, but not in IL-1 knockout mice. J Neuroinflamm 10: 143, 2013.

5. Tang Y and Le W: Differential roles of M1 and M2 microglia in neurodegenerative diseases. Mol Neurobiol 53: 1181-1194, 2016.

6. Varnum MM and Ikezu T: The classification of microglial activation phenotypes on neurodegeneration and regeneration in Alzheimer's disease brain. Arch Immunol Ther Exp (Warsz) 60: 251-266, 2012.

7. Kim BW, Koppula S, Kumar H, Park JY, Kim IW, More SV, Kim IS, Han SD, Kim SK, Yoon SH and Choi DK: $\alpha$-Asarone attenuates microglia-mediated neuroinflammation by inhibiting NF kappa B activation and mitigates MPTP-induced behavioral deficits in a mouse model of Parkinson's disease.. Neuropharmacology 97: 46-57, 2015.

8. Rubin LL and de Sauvage FJ: Targeting the Hedgehog pathway in cancer. Nat Rev Drug Discov 5: 1026-1033, 2006.

9. de Sauvage F: The HH signaling pathway in cancer. Bull Mem Acad R Med Belg 162: 219-223, 2007.

10. Banerjee SB, Rajendran R, Dias BG, Ladiwala U, Tole S and Vaidya VA: Recruitment of the Sonic hedgehog signaling cascade in electroconvulsive seizure-mediated regulation of adult rat hippocampal neurogenesis. Eur J Neurosci 22: 1570-1580, 2005.

11. Sims JR, Lee SW, Topalkara K, Qiu J, Xu J, Zhou Z and Moskowitz MA: Sonic hedgehog regulates ischemia/hypoxia-induced neural progenitor proliferation. Stroke 40: 3618-3636, 2009

12. Hung HC, Hsiao YH and Gean PW: Sonic hedgehog signaling regulates amygdalar and extinction of fear memory. Eur Neuropsychopharmacol 25: 1723-1732, 2015.

13. Hung HC, Hsiao YH and Gean PW: Learning induces sonic hedgehog signaling in the amygdala which promotes neurogenesis and long-term memory formation. Int J Neuropsychopharmacol 18: pyu071, 2015.

14. Dass B, Iravani MM, Jackson MJ, Engber TM, Galdes A and Jenner P: Behavioural and immunohistochemical changes following supranigral administration of sonic hedgehog in 1-methyl-4-phenyl-1,2,3,6-tetrahydropyridine-treated common marmosets. Neuroscience 114: 99-109, 2002.

15. Zhang Y, Dong W, Guo S, Zhao S, He S, Zhang L, Tang Y and Wang $\mathrm{H}$ : Lentivirus-mediated delivery of sonic hedgehog into the striatum stimulates neuroregeneration in a rat model of Parkinson disease. Neurol Sci 35: 1931-1940, 2014.

16. Schabbauer G, Tencati M, Pedersen B, Pawlinski R and Mackman N: PI3K-Akt pathway suppresses coagulation and inflammation in endotoxemic mice. Arterioscler Thromb Vasc Biol 24: 1963-1969, 2004

17. Kim SN, Kim ST, Doo AR, Park JY, Moon W, Chae Y, Yin CS, Lee $\mathrm{H}$ and Park HJ: Phosphatidylinositol 3-kinase/Akt signaling pathway mediates acupuncture-induced dopaminergic neuron protection and motor function improvement in a mouse model of Parkinson's disease. Int J Neurosci 121: 562-569, 2011.

18. Zhao Y, Zhang Q, Xi J, Xiao B, Li Y and Ma C: Neuroprotective effect of fasudil on inflammation through PI3K/Akt and Wnt/ $\beta$-catenin dependent pathways in a mice model of Parkinson's disease. Int J Clin Exp Pathol 8: 2354-2364, 2015.

19. Dai R, Xia Y, Mao L, Mei Y, Xue Y and Hu B: Involvement of PI3K/Akt pathway in the neuroprotective effect of Sonic hedgehog on cortical neurons underoxidative stress. J Huazhong Univ Sci Technolog Med Sci 32: 856-860, 2012.

20. Xia YP, Dai RL, Li YN, Mao L, Xue YM, He QW, Huang M, Huang Y, Mei YW and $\mathrm{Hu}$ B: The protective effect of sonic hedgehog is mediated by the phosphoinositide [corrected] 3-kinase/AKT/Bcl-2 pathway in cultured rat astrocytes under oxidative stress. Neuroscience 209: 1-11, 2012.

21. Guo Z, Xu S, Du N, Liu J, Huang Y and Han M:Neuroprotective effects of stemazole in the MPTP induced acute model of Parkinson's disease: Involvement of the dopamine system. Neurosci Lett 616: 152-159, 2016.

22. Kuribara H, Higuchi Y and Tadokoro S: Effects of central depressants on rota-rod and traction performances in mice. Jpn J Pharmacol 27: 117-126, 1977.

23. Ouchi Y, Yoshikawa E, Sekine Y, Futatsubashi M, Kanno T, Ogusu T and Torizuka T: Microglial activation and dopamine terminal lossin early Parkinson's disease. Ann Neurol 57: 168-75, 2005.

24. Ouchi Y, Yagi S, Yokokura $M$ and Sakamoto $M$ : Neuroinflammation in the living brain of Parkinson's disease. Parkinsonism Relat Disord 15 (Suppl 3): S200-S204, 2009. 
25. Cappellano G, Carecchio M, Fleetwood T, Magistrelli L, Cantello R, Dianzani U and Cormi C: Immunity and inflammation in neurodegenerative disease. Am J Neurodegener Dis 21: 89-107, 2013

26. Gonzalez-Reyes LE, Verbitsky M, Blesa J, Jackson-Lewis V Paredes D, Tillack K, Phani S, Kramer ER, Przedborski S and Kottmann AH: Sonic hedgehog maintains cellular and neurochemical homeostasis in the adult nigrostriatal circuit. Neuron 75: 306-319, 2012.

27. Gordon R, Singh N, Lawana V, Ghosh A, Harischandra DS Jin H, Hogan C, Sarkar S, Rokad D, Panicker N, et al: Protein kinase Cd upregulation in microglia drives neuroinflammatory responses and dopaminergic neurodegeneration in experimental models of Parkinson's disease. Neurobiol Dis 93: 96-114, 2016.

28. Kim BW, Koppula S, Park SY, Kim YS, Park PJ, Lim JH, Kim IS and Choi DK: Attenuation of neuroinflammatory responses and behavioral deficits by Ligusticum officinale (Makino) Kitag in stimulated microglia and MPTP-induced mouse model of Parkinson's disease. J Ethnopharmacol 164: 388-397, 2015.

29. Zhou X, Liu Z, Jang F, Xiang C, Li Y and He Y: Autocrine Sonic hedgehog attenuates inflammation in cerulein-induced acute pancreatitis in mice via upregulation of IL-10. PLoS One 7: e44121, 2012.
30. Haas SJ, Zhou X, Machado V, Wree A, Krieglstein K and Spittau B: Expression of Tgf $\beta 1$ and inflammatory markers in the 6-hydroxydopamine mouse model of Parkinson's disease. Front Mol Neurosci 9: 7, 2016.

31. Quesada A, Lee BY and Micevych PE: PI3 kinase/Akt activation mediates estrogen and IGF-1 nigral DA neuronal neuroprotection against a unilateral rat model of Parkinson's disease. Dev Neurobiol 68: 632-644, 2008

32. Kim CH, Han BS, Moon J, Kim DJ, Shin J, Rajan S, Nguyen QT, Sohn M, Kim WG, Han M, et al: Nuclear receptor Nurrl agonists enhance its dual functions and improve behavioral deficits in an animal model of Parkinson's disease. Proc Natl Acad Sci USA 112: 8756-8761, 2015.

33. Maguire-Zeiss KA and Federoff HJ: Future directions for immune modulation in neurodegenerative disorders: Focus on Parkinson's disease. J Neural Transm (Vienna) 117: 1019-1025, 2010. 